\title{
Power systems simulation using ontologies to enable the interoperability of multi-agent systems
}

\author{
Gabriel Santos, Francisco Silva, Brígida Teixeira, Zita Vale \\ GECAD Research Group, \\ Polytechnic of Porto, Portugal \\ \{gajls, fspsa, bccta, zav\}@isep.ipp.pt
}

\author{
Tiago Pinto \\ Bisite Research Group, \\ University of Salamanca, Spain \\ tpinto@usal.es
}

\begin{abstract}
A key challenge in the power and energy field is the development of decision-support systems that enable studying big problems as a whole. The interoperability between systems that address specific parts of the global problem is essential. Ontologies ease the interoperability between heterogeneous systems providing semantic meaning to the information exchanged between the various parties. The use of ontologies within Smart Grids has been proposed based on the Common Information Model, which defines a common vocabulary describing the basic components used in electricity transportation and distribution. However, these ontologies are focused on utilities needs. The development of ontologies that allow the representation of diverse knowledge sources is essential, aiming at supporting the interaction between entities of different natures, facilitating the interoperability between these systems. This paper proposes a set of ontologies to enable the interoperability between different types of simulators, namely regarding electricity markets,the smart grid, and residential energy management. A case study based on real data shows the advantages of the proposed approach in enabling comprehensive power system simulation studies.
\end{abstract}

Index Terms-Multi-agent Simulation, Power and Energy Systems, Semantic interoperability.

\section{INTRODUCTION}

The electricity markets (EM) paradigm has been changing over the last decades due to its restructuring. On one hand, transformations were applied, such as the privatization, liberalization and international integration of previously nationally owned systems [1]. On the other hand, the high penetration and investments in renewable energies sources (RES) to increase energy efficiency play an important role in the reduction of the greenhouse gas emissions, reducing the dependency on limited fossil fuels [2]. Portugal has also been investing on RES [3], in the last years, to be in accordance with the recent changes in the European Union legislation to increase energy efficiency [4].

Nowadays, EM operation uses more complex and trustworthy models. However they are still restricted to the participation of large players [5]. This problem is being addressed

This work has received funding from the European Union's Horizon 2020 research and innovation programme under the Marie Sklodowska-Curie grant agreement No 641794 (project DREAM-GO) and from FEDER Funds through COMPETE program and from National Funds through FCT under the project UID/EEA/00760/2013. differently all around the world. Still, some common solutions are being adopted in last years. Some EM have evolved to regional and, in some cases, continental scale markets, supporting the transactions of large amounts of electrical energy while enabling the efficient use of RES where it exceeds the local needs.

In the smart grids (SG) and microgrids (MG) context, new opportunities emerge. The consumer becomes a prosumer, being able to adapt his consumption to his own production, while selling the surplus power, when available, or buying the demand required, when needed [6]. This new paradigm gives the consumer an active role in the grid environment, contributing to a change in his own use habits due to the intensive use of RES of intermittent nature, which affects the balance between consumption and production [7]. In this context, smart homes should consider three important elements: a communication network, intelligent control systems, and home automation [8]. Moreover, smart home management systems should be able to manage consumption and production effectively, as well as the participation in Demand Response (DR) events, such as dynamic energy tariffs [9]. It should also consider complex environments connected to both smart devices and electrical appliances, providing different smart functions and contextaware services to the end-user [10].

Due to this constant evolution in EM environment, it is essential for professionals in the area to completely understand the emerging markets' models and principles in order to evaluate their investments and strategies under such a competitive environment. The use of simulation tools has grown to provide professionals with the right tools for understanding those mechanisms and how the involved players' interaction affects the markets' outcomes. Although several tools have arisen to satisfy the different markets' requirements, the need for the integration of different market models and platforms brings out the lack of interoperability between heterogeneous simulation tools. Simulation platforms could benefit from sharing their knowledge and market models with each other. Such tools would provide the means for an actual improvement in current EM studies and development by allowing joint and hybrid simulations of distinct market models, as well as more complex simulation scenarios [11]. The integration of distinct market models and platforms issues the need for communication capabilities that allow heterogeneous entities (such as software 
agents or web services) to understand each other and cooperate toward a common goal. An effective communication language is needed, considering complex functionalities and strategies, as well as several types of energy resources, in the context of SG and MG [12].

Ontologies define a common "language" that can be interpreted and understood by any software or software agent [13], allowing heterogeneous systems to coexist and collaborate [14].

This work proposes a solution to enable the interoperability between heterogeneous multi-agent systems (MAS) directed to the study and analysis of EM, SG and house energy management systems. To this end, this paper proposes the development of a society of MAS aimed at the simulation and study of energy systems, taking advantage of existing simulation and decision-support tools, namely: the MultiAgent Simulator of Competitive Electricity Markets (MASCEM) [15], [16], Adaptive Decision Support for Electricity Markets Negotiations (AiD-EM) [17], Multi-Agent Smart Grid Platform (MASGriP) [18], [19] and SCADA House Intelligent Management (SHIM) [20].

The next section overviews the related work on multi-agent simulation applied to EM and power systems. Section III presents the main contribution of this work, i.e., ontologies for power systems simulation. Section IV illustrates an example case study. Finally, section $\mathrm{V}$ features the most relevant conclusions.

\section{MUlti-AGENT SIMUlATION OF POWER SYSTEMS}

MAS are not necessarily simulation platforms but simulations are essential for EM and SG study, namely concerning scenarios comparison, future evolution study and sensitive analysis. Multi-agent simulation combined with artificial intelligence techniques may result in sophisticated tools, namely in players and markets modelling, strategic bidding and decisionsupport in the power and energy systems scope [21]. Several works sustain the adequacy and ability of MAS simulation in this area [15], [17], [19], [22], [23], [24]. In order to provide players with adequate tools to adapt themselves to the new reality, gaining experience to act in the frame of a changing economic, financial, and regulatory environment, EM and power systems simulators must be able to cope with an evolving complex and dynamic reality [25]. Multi-agent simulation tools enable the ease enlargement of models while accomplishing with the markets' future evolution. Two of the best advantages of multi-agent based platforms are the integration of new models and the interconnection with other systems, with their own social environments.

\section{A. MASCEM}

MASCEM [15], [16] is a modelling and simulation tool. It has been developed with the goal of studying the operation of the restructured, complex and competitive EM by modelling the main market entities, such as: the market and system operators, buyer and seller agents; and their interactions.

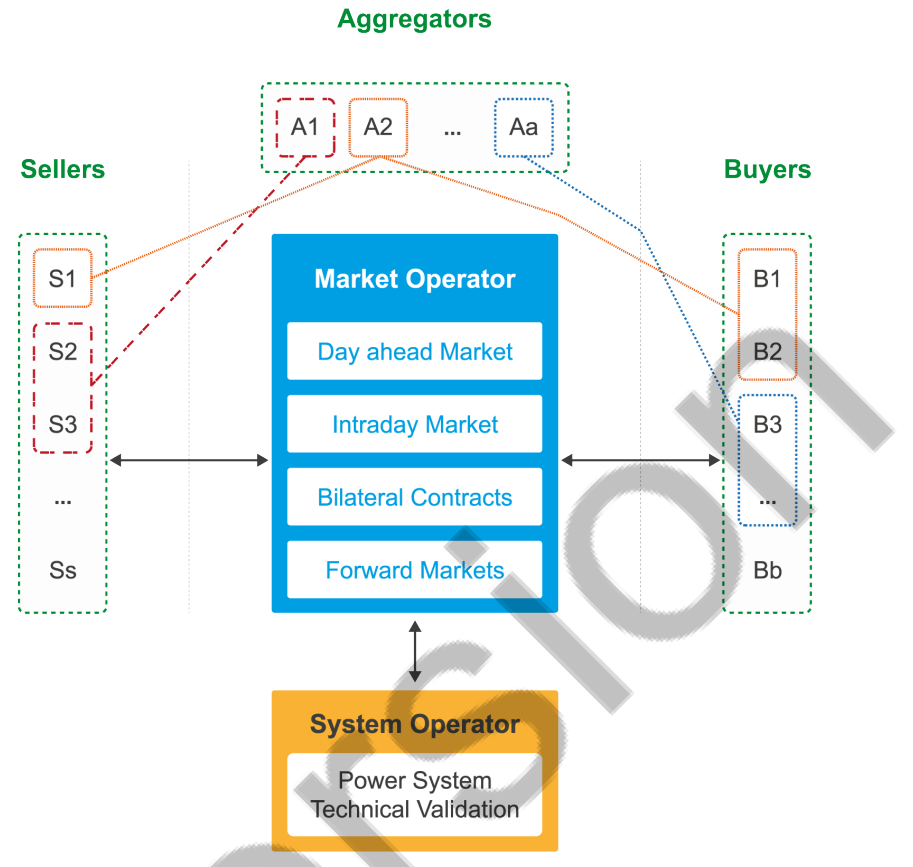

Figure 1. MASCEM's multi-agent model, adapted from [26]

MASCEM's main goal is to be used as a decision support tool for short/medium-term purposes and also for long-term decisions, as the ones taken by market regulators. To this end, it must simulate as many market models, players and operators as possible in order to emulate the real EM operation.

The simulation of different market types is considered, namely: day-ahead and intraday pools (asymmetric or symmetric, with or without complex conditions), bilateral contracts and forward markets. By selecting a combination of the available market models, hybrid markets' simulation is also available.

Figure 1 illustrates MASCEM's multi-agent model.

To support players decisions in accordance with their characteristics and goals, medium/long-term gathering of data and experience is also considered.

\section{B. MASGriP}

MASGriP [19] is a multi-agent modelling and simulation tool, proposing a set of possible coalitions for the management and operation of SG, considering all the typically involved players, which are also modelled by software agents. The considered entities are different types of operators, aggregators, and several energy resources, such as several types of consumers (e.g. industrial, commercial, residential), different types of producers (e.g. wind farms, solar plants, cogeneration units), electric vehicles with vehicle-to-grid capabilities, among others.

Some of MASGriP's agents may be directly connected to physical installations, allowing the automatic management and control of the respective resources. The physical installations are, more specifically, GECAD's three campus buildings, and a controlled laboratory house: SHIM [20]. 


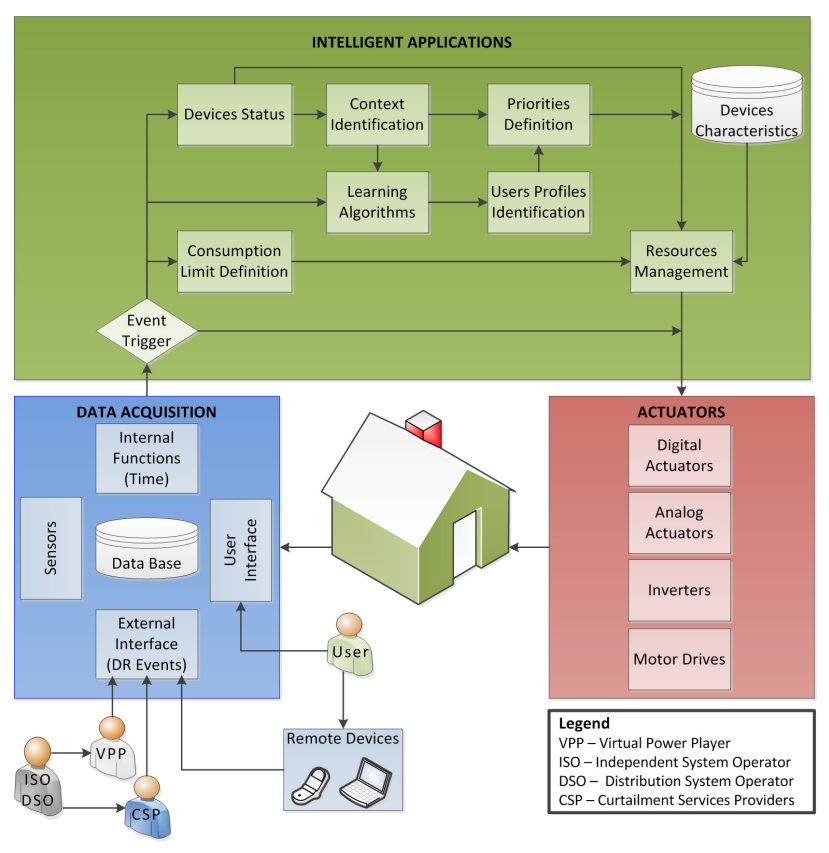

Figure 2. SHIM simulation platform [27]

MASGriP takes advantage of several intelligent methodologies to support agents operation at different levels. It also uses real-time simulation to complement simulations with the analysis of the methods impact in the network lines [19].

\section{SHIM}

SHIM [20], [27] is a simulation tool in the context of intelligent house management with the main goal of testing, simulating, and validating new algorithms and methodologies. It is composed of different modules: the Data acquisition, the Actuators, and the Intelligent Applications. SHIM platform and the structure used for house management are better detailed in [27]. Figure 2 illustrates SHIM's platform structure.

The resources management block, of the Intelligent Applications module, is responsible for managing energy consumption in a domestic consumer. This module is activated by an event trigger. The first step collects all the needed data for the current context, i.e. the resources' features and priority, power consumption limits, and the actual status of each resource (e.g. refrigerator, HVAC, lights, etc.).

The impact of loads curtailment depend on the loads and also on the energy resources management (ERM) status. To try to minimize this impact an optimization algorithm is used. A priority value between 0 and 10 is used to classify the resources, being 10 the lowest priority and 0 the highest. Detailed information on the optimization method is available at [27].

\section{ONTOLOGIES FOR POWER SYSTEMS SIMULATION}

One of the main advantages of developing multi-agent systems is the easily integration with heterogeneously developed agent-based systems, by communicating in a common language and vocabulary [14]. The increasing application of
MAS technology within power and energy systems, promotes the use of standards to enable understandable communications between heterogeneous systems [28].

Currently, MAS developed for the power and energy systems domain use their own specific and private ontologies. These ontologies share common concepts differently represented, and automatic translations between them are not as straightforward as it may seem. On the other hand, manual mappings between them increases the human effort and cost of implementation significantly, once every time an ontology changes, all related mappings must be verified and corrected, if necessary.

Trying to overcome this issue, [28] suggests the use of an upper ontology, which gathers general and abstract concepts of the domain, to insure a common basis for the representation of more specific concepts and their relations, and to reduce the complexity of ontology mapping. Ontologies provide semantic meaning to the information exchanged between the different parties, facilitating interoperability between heterogeneous systems [28].

\section{A. Electricity Markets Ontology}

The use of semantics for heterogeneous systems' interoperability enables full knowledge exchange, taking advantage of the functionalities made available by each system. To enable interoperability between EM agent-based systems the authors' have proposed the Electricity Markets Ontology (EMO) [29]. EMO includes abstract concepts and axioms representing the main existing EM. It tries to be as inclusive as possible in order to be reused and/or extended in the development of EM specific ontologies.

To enable semantic communications using EMO concepts, two additional modules have been developed separately [11]: the Call for Proposal (CFP); and the Electricity Markets Results (EMR) ontologies. These modules define Requests, Responses and Informs, enabling a semantic interaction between the participating software agents. EMO, CFP and EMR are publicly available ${ }^{1}$ so they can be (re)used by third-party developers in the context of the wholesale EM. More details about EMO, CFP and EMR can be found in [11], [29].

\section{B. Semantics for Smart Grids context}

There are in literature some proposals for the use of ontologies in the scope of SG [30], [31]. An important requirement for the interoperability between heterogeneous systems is to reuse existing semantics [32]. However, these ontologies are only focused on the utilities needs since are all based on the Common Information Model (CIM). CIM defines a common vocabulary that describes the basic components used in electricity transportation and distribution.

It is essential to develop ontologies that enable the representation of different knowledge sources, to support the interactions between heterogeneous entities of distinct natures, facilitating interoperability among them.

\footnotetext{
${ }^{1}$ http://www.mascem.gecad.isep.ipp.pt/ontologies/
} 
The ITEA2 project Smart Energy Aware Systems (SEAS) ${ }^{2}$, which has received the ITEA2 Award of Excellence 2017, developed SEAS Knowledge Model as a basis for semantic interoperability in SEAS environments [33]. SEAS project "aimed at designing and developing a global ecosystem of services and smart things collectively capable of ensuring the stability and the energy efficiency of the future energy grid" [34]. Sub-chapter III-C overviews the authors' contribution to the SEAS knowledge model, regarding the ISEP $^{3}$ Campus Microgrid management, control and simulation.

\section{Ontologies for communications and knowledge represen- tation}

Ontologies provide the means to successfully exchange meaningful information that can be easily interpreted by software agents. On the other hand, using a reasoner, ontologies also enable to infer knowledge from the gathered information. Ontologies are used to enable semantic interoperability between heterogeneous agents and/or agent-based platforms of the Campus Microgrid. The developed ontologies are useful not only for communication purposes, but also for knowledge representation and sharing among the software agents.

Regarding SEAS knowledge model (http://w3id.org/seas/), GECADs contribution included the discussion and developing of the following ontology modules:

- SEAS-ActorOntology: The SEAS Actor Ontology for the ITEA2 SEAS project (http://w3id.org/seas/ ActorOntology);

- SEAS-ActorVocabulary: The Seas Actor Vocabulary defined for the ITEA2 SEAS project (http://w3id.org/seas/ ActorVocabulary);

- SEAS-AreaOntology: This ontology is targeted for defining structure of buildings (or more general facilities) and zones related to control and measurement with links to various BIM (Building Information Model) related standards (http://w3id.org/seas/AreaOntology);

- SEAS-BuildingCategories Vocabulary: The Seas Building Categories for the ITEA2 SEAS (Smart Energy Aware Systems) project. This module presents subcategories for classifying building related concepts (http://w3id.org/ seas/BuildingCategoriesVocabulary);

- SEAS-EnergyFormVocabulary: This vocabulary defines forms of energy, such as ElectricEnergy, NuclearEnergy, MagneticEnergy or ThermalEnergy (http://w3id.org/seas/ EnergyFormVocabulary);

- SEAS-ElectricityPlayerOntology: This ontology defines electricity players and electricity market, as systems that exchange electricity (http://w3id.org/seas/ ElectricityPlayerOntology);

\footnotetext{
${ }^{2}$ https://www.the-smart-energy.com

${ }^{3}$ Institute of Engineering - Polytechnic of Porto. http://isep.ipp.pt
}

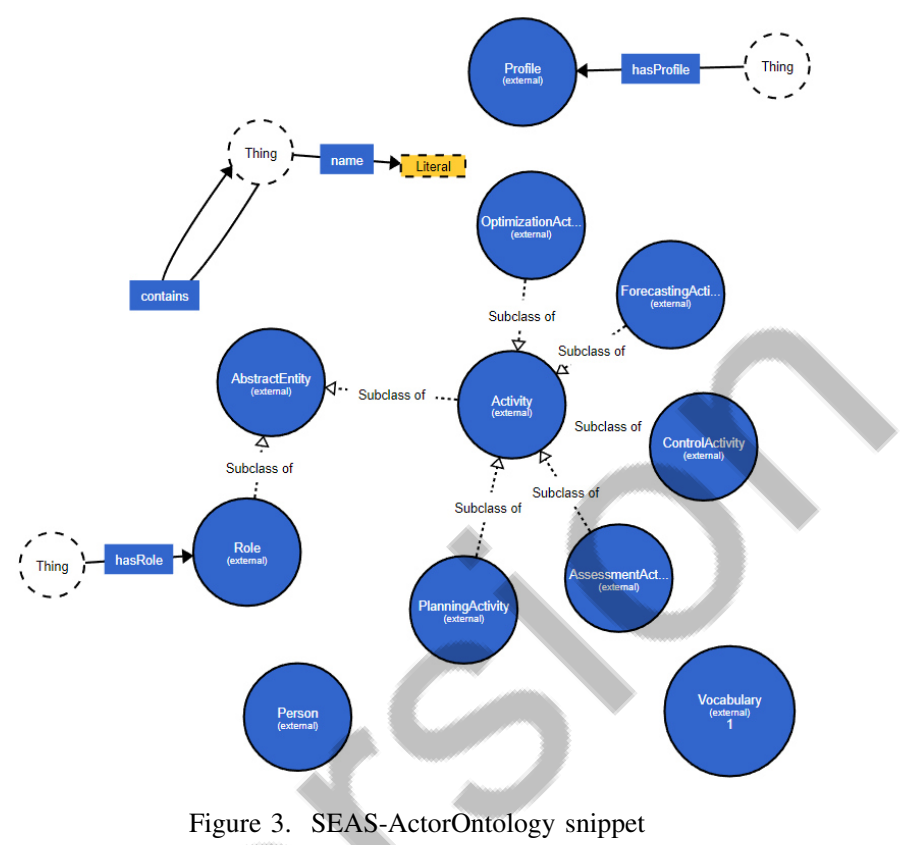

- SEAS-ElectricPowerSystemVocabulary: The SEAS Electric Power System Vocabulary defines: 1) Electric power systems that consume, produce, or store electricity, 2) electrical connections between electric power systems, where electricity is exchanged, and 3) electrical connection Points of electric power systems, through which electricity flows in/out the power systems (http://w3id. org/seas/ElectricPowerSystemVocabulary);

- SEAS-FlexibilityVocabulary: The SEAS Flexibility Vocabulary defines code lists to interpret evaluations of operating features of interest. For instance, the value may be the minimal operating value (http://w3id.org/seas/ FlexibilityVocabulary);

- SEAS-LightSystemOntology: This vocabulary defines light Systems, and their common properties (http://w3id. org/seas/LightSystemOntology);

- SEAS-ThermodynamicSystemOntology: This ontology is targeted for defining thermodynamic systems and their relations (http://w3id.org/seas/ ThermodynamicSystemOntology);

- SEAS-ThermodynamicSystemVocabulary: This vocabulary defines common properties of thermodynamic systems, and evaluation interpretation code lists (http://w3id. org/seas/ThermodynamicSystemVocabulary).

Figure 3 presents a snippet of the SEAS-ActorOntology. This image has been chosen as an illustrative example due to its limited size and for an easier understanding. The length of the SEAS ontology is too extensive to be fully shown in this document. The SEAS knowledge model is addressed in detail in [33]. 


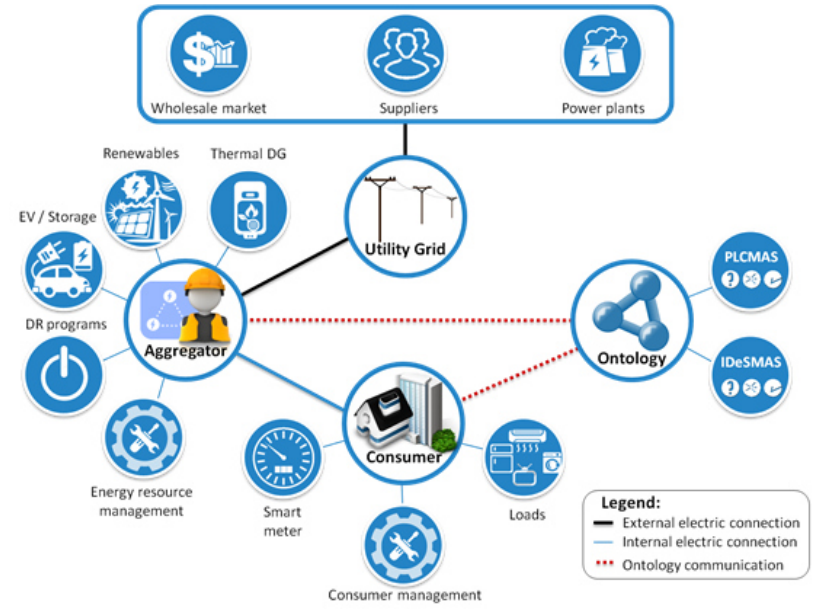

Figure 4. Case study scenario

It should be noticed that besides the ontology modules listed above, the Campus Microgrid also reuses other SEAS modules developed with the contribution of other partners.

The developed ontologies not only enable the interoperability between different MAS but also represent the concepts needed to understand and use real data, from different sources. These data can be acquired in real time through analysers/sensors, or even databases available online. For that, the developed ontologies allow the representation of knowledge in a common vocabulary, regardless of the source; thus facilitating interoperability between the various heterogeneous systems and data, information and knowledge sources, with the ultimate goal of achieving an enhanced simulation platform for fully transactive energy systems.

\section{CASE Study}

The present case study considers the joint simulation of the interaction between SHIM (for residential energy management), MASGriP (for the management of energy resources in a SG environment), and MASCEM (for EM simulation). A SG simulation is executed, in which the SG operator manages the associated resources, taking into account the interaction with the several consumers and the EM. The resources scheduling that result from the SG energy management and its participation in the market, is then communicated to the consumers, which apply their own energy resources management to manage their loads. The management of a particular house is reflected in a real laboratory house in GECAD facilities. Figure 4 illustrates this case study scenario.

A 25-bus microgrid is considered. It includes 90 loads, 17 photovoltaic (PV) systems, 17 storage systems and 5 external suppliers. And it considers four types of consumers, namely: 7 Residential Houses, 8 Residential Buildings, 2 Office Buildings, and 1 Residential Heat Pump.

The simulation is based on real data sets and some loads are monitored in real time, such as Load 1 (GECAD office building), Load 6 (GECAD smart home lab), and Load 4, 7-9 (residential consumers, from a german partner, monitored

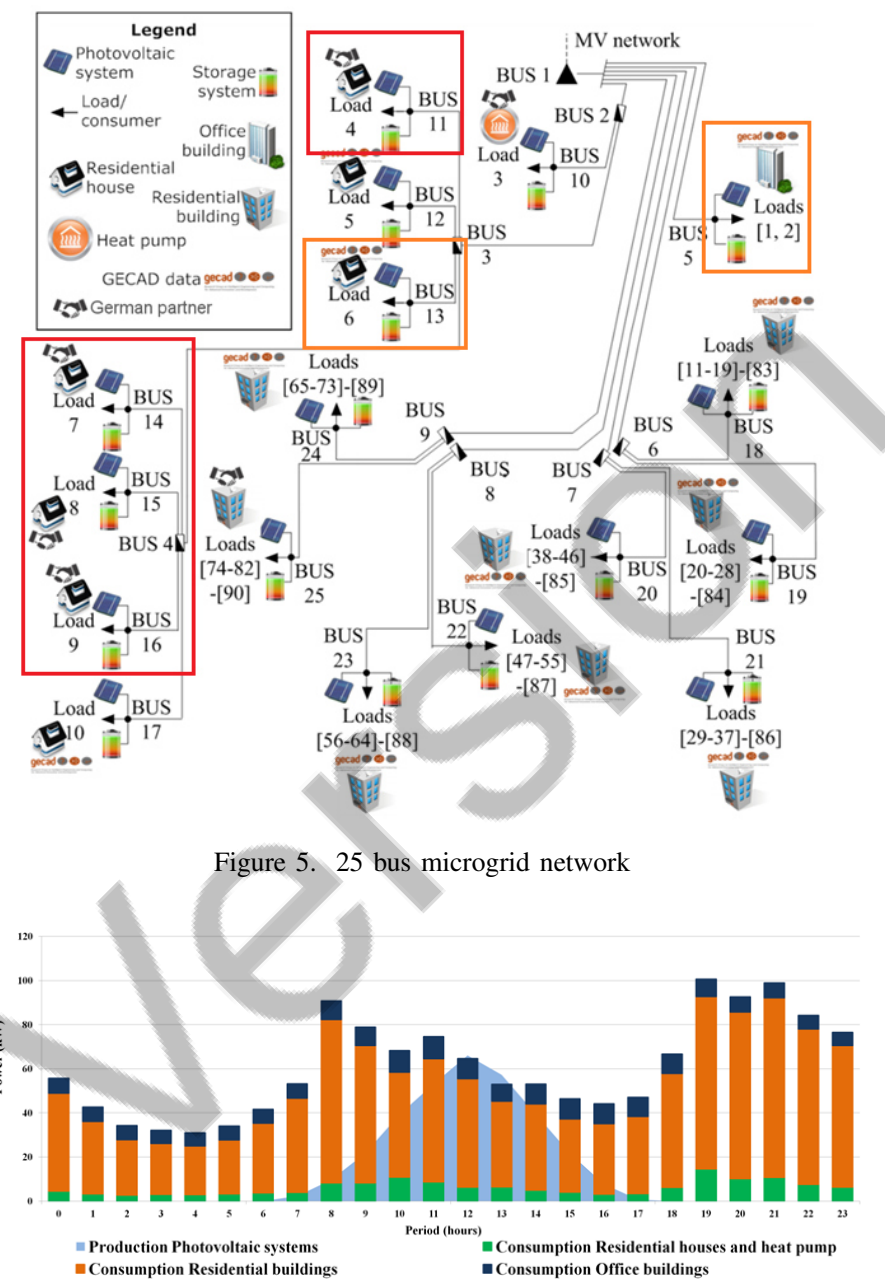

Figure 6. Demand and PV generation forecast

in real time). Figure 5 presents the considered network and respective loads.

The considered simulation day is the $22^{\text {nd }}$ of January, 2013. For this particular day, the demand and PV generation forecast is shown in Figure 6. It can be seen that the peak loads are expected in the morning and at night periods (over than 60 $\mathrm{kW})$.

The simulations starts with the aggregator executing the energy resources management (ERM) scheduling for the next day, considering the estimated forecasts, and considering the participation in the wholesale market. The participation in the EM is simulated using MASCEM, where the aggregator will be able to buy energy in periods where there is not enough generation, or to sell it in the periods where the generation exceeds the needs from the microgrid. Figure 7 illustrates the 25 bus network ERM scheduling for the day-ahead horizon.

After, the aggregator communicates to each aggregated player the ERM scheduling results for their specific consumption. Then, each aggregated player executes their own SHIM optimization in order to comply with the resources scheduling sent by the aggregator. For this case study purposes, we will only analyse Load 1 (GECAD office building). 


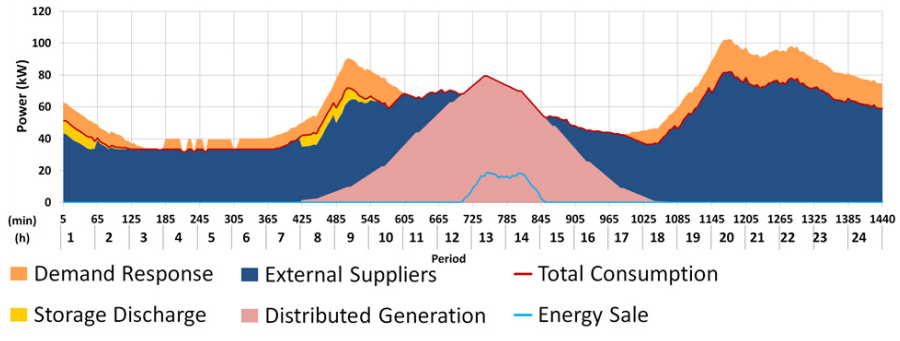

Figure 7. ERM scheduling for the day-ahead

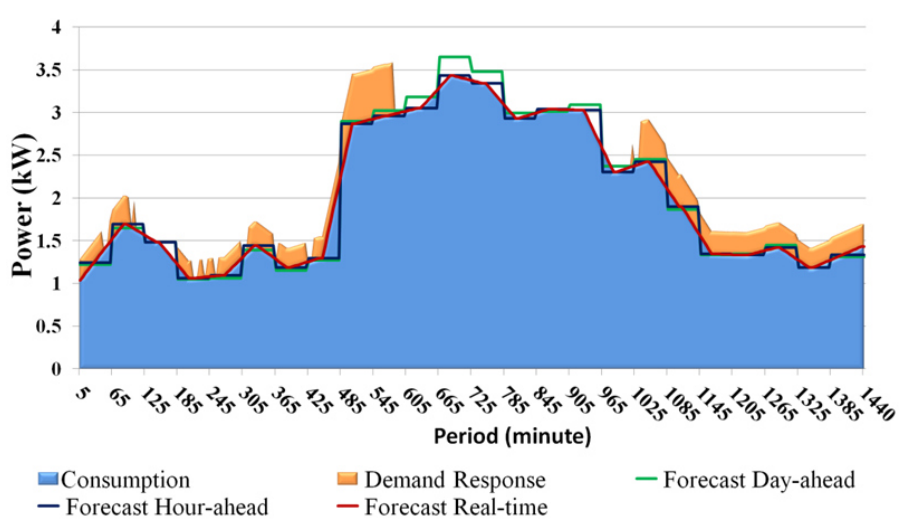

Figure 8. ERM scheduling for Load 1 (GECAD office building)

The aggregator's ERM scheduling result for this building is presented in Figure 8.

Given the scheduling sent by the aggregator, player Load 1 executes the SHIM tool to perform a local scheduling of the building, trying to optimize their resources and energy consumption for that day. Figure 9 shows the local scheduling results for player Load 1.

The SHIM tool determines the scheduling results for each load according to the limits indicated by the aggregator. As can be seen some demand response is scheduled for periods of the day when the generatin is lower. The local energy resources scheduling using SHIM needs to accomodate these, taking into account the specific needs and characteristics of the house. The limit of comsumption is achieved by the difference between

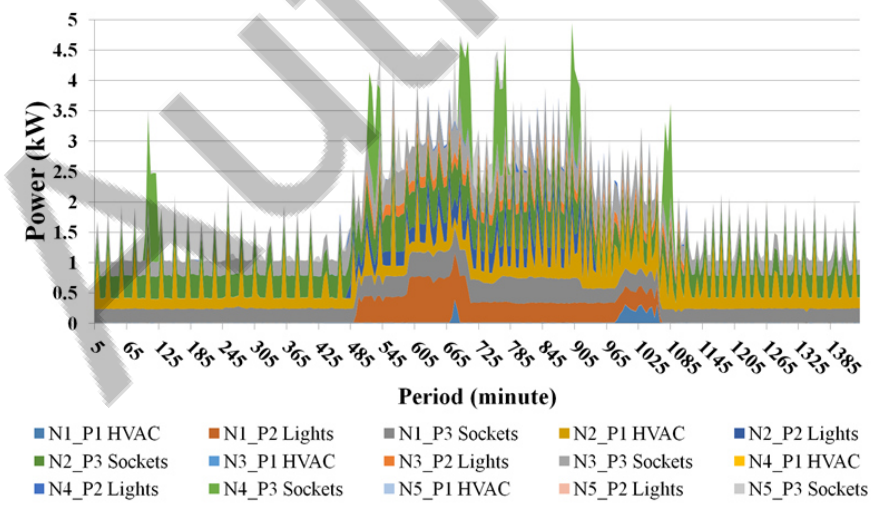

Figure 9. SHIM scheduling for Load 1 (GECAD office building) consumption and DR. SHIM tool also enables the automatic load control according to the obtained results, in order to execute the respective scheduling.

Considering this winter scenario, resources like HVAC and water heater, have higher priority in the optimization. If this was a summer scenario, different context would been taken into account, such as updating the resources' priorities in SHIM tool; e.g. the water heater would have lower priority as well as the lights, since there are more hours with luminosity. On the other hand, the ERM of the aggregator would also consider more sun hours, which would reflect in higher power generation by PV systems.

\section{CONCLUSIONS}

This paper presents an innovative and advanced approach to enable multi-agent system interoperability in the power and energy systems domain. To achieve such interoperability, the use of ontologies is considered. Ontologies enable the interoperability between heterogeneous systems in the communication level.

The multi-agent simulation platforms integrated go from EM operation (MASCEM), to aggregators, and respective aggregated players (e.g. consumers, producers, and prossumers), with the intention of showing the ability to simulate more complex and complete scenarios by taking advantage of semantic interoperability. The ontologies used are introduced in Section III, where is made a brief description of each module.

The results of the presented case study show that the several agents are able to communicate effectively by using the proposed semantics. The use of ontologies enabled an appropriate interaction between the software agents, as well as with the several algorithms and operational levels. In this way, a full communication flow is accomplished from the SG operator to the end user, and also from the aggregator to the EM operator. This allows all information and intelligent modules to improve results on the entire chain. The final and main contribution from the proposed approach is to enable comprehensive simulations of the power and energy system, composed by multiple heterogeneous systems, algorithms, tools and physical devices, thus allowing a more realistic and complete study of the power system domain.

\section{REFERENCES}

[1] K. C. Sharma, R. Bhakar, and H. Tiwari, "Strategic bidding for wind power producers in electricity markets," Energy Conversion and Management, vol. 86, no. Supplement C, pp. 259 - 267, 2014. [Online]. Available: http://www.sciencedirect.com/science/article/ pii/S0196890414004129

[2] R. Wstenhagen and E. Menichetti, "Strategic choices for renewable energy investment: Conceptual framework and opportunities for further research," Energy Policy, vol. 40, no. Supplement C, pp. 1 - 10, 2012, strategic Choices for Renewable Energy Investment. [Online]. Available: http://www.sciencedirect.com/science/article/pii/S0301421511005064

[3] L. Fernandes and P. Ferreira, "Renewable energy scenarios in the portuguese electricity system," Energy, vol. 69, no. Supplement C, pp. 51 - 57, 2014. [Online]. Available: http://www.sciencedirect.com/ science/article/pii/S036054421400245X

[4] Eurostat, "Smarter, greener, more inclusive? indicators to support the europe 2020 strategy," http://ec.europa.eu/eurostat/de/web/ products-statistical-books/-/KS-EZ-14-001, 2015, accessed: 2017-0928. 
[5] L. Meeus, K. Purchala, and R. Belmans, "Development of the internal electricity market in europe," The Electricity Journal, vol. 18, no. 6, pp. 25-35, 2005. [Online]. Available: http://EconPapers.repec.org/RePEc: eee:jelect:v:18:y:2005:i:6:p:25-35

[6] G. Brusco, A. Burgio, D. Menniti, A. Pinnarelli, and N. Sorrentino, "Energy management system for an energy district with demand response availability," IEEE Transactions on Smart Grid, vol. 5, no. 5, pp. 23852393, Sept 2014.

[7] J. M. Guerrero, F. Blaabjerg, T. Zhelev, K. Hemmes, E. Monmasson, S. Jemei, M. P. Comech, R. Granadino, and J. I. Frau, "Distributed generation: Toward a new energy paradigm," IEEE Industrial Electronics Magazine, vol. 4, no. 1, pp. 52-64, March 2010.

[8] J. Ye, Q. Xie, Y. Xiahou, and C. Wang, "The research of an adaptive smart home system," in 2012 7th International Conference on Computer Science Education (ICCSE), July 2012, pp. 882-887.

[9] P. Faria and Z. Vale, "Demand response in electrical energy supply: An optimal real time pricing approach," Energy, vol. 36, no. 8, pp. 5374 - 5384, 2011, pRES 2010. [Online]. Available: http://www.sciencedirect.com/science/article/pii/S036054421100435X

[10] J. y. Son, J. h. Park, K. d. Moon, and Y. h. Lee, "Resource-aware smart home management system by constructing resource relation graph," IEEE Transactions on Consumer Electronics, vol. 57, no. 3, pp. 11121119, August 2011.

[11] G. Santos, T. Pinto, I. Praa, and Z. Vale, "An interoperable approach for energy systems simulation: Electricity market participation ontologies," Energies, vol. 9, no. 11, p. 878, 2016. [Online]. Available: http://www.mdpi.com/1996-1073/9/11/878

[12] G. Santos, F. Femandes, T. Pinto, M. Silva, O. Abrishambaf, H. Morais, and Z. Vale, "House management system with real and virtual resources: Energy efficiency in residential microgrid," in 2016 Global Information Infrastructure and Networking Symposium (GIIS), Oct 2016, pp. 1-6.

[13] M. FRIKHA, M. MHIRI, and F. GARGOURI, "A semantic social recommender system using ontologies based approach for tunisian tourism," ADCAIJ: Advances in Distributed Computing and Artificial Intelligence Journal, vol. 4, no. 1, 2015. [Online]. Available: http:// revistas.usal.es/index.php/2255-2863/article/view/ADCAIJ20154190106

[14] "Foundation for Intelligent Physical Agents (FIPA), FIPA Ontology Service Specification," Available: http://www.fipa.org/specs/fipa00086/ XC00086D.html, 2001, accessed: 2017-05-16.

[15] G. Santos, T. Pinto, I. Praa, and Z. Vale, "Mascem: Optimizing the performance of a multi-agent system," Energy, vol. 111, pp. 513 - 524, 2016. [Online]. Available: http://www.sciencedirect.com/science/article/ pii/S0360544216307654

[16] I. Praça, C. Ramos, Z. Vale, and M. Cordeiro, "Mascem: a multiagent system that simulates competitive electricity markets," IEEE Intelligent Systems, vol. 18, no. 6, pp. 54-60, Nov 2003.

[17] T. Pinto, H. Morais, T. M. Sousa, T. Sousa, Z. Vale, I. Praça, R. Faia, and E. J. S. Pires, "Adaptive portfolio optimization for multiple electricity markets participation," IEEE Transactions on Neural Networks and Learning Systems, vol. 27, no. 8, pp. 1720-1733, Aug 2016.

[18] P. Oliveira, T. Pinto, H. Morais, and Z. Vale, "Masgrip - a multi-agent smart grid simulation platform," in 2012 IEEE Power and Energy Society General Meeting, July 2012, pp. 1-8.

[19] L. Gomes, P. Faria, H. Morais, Z. Vale, and C. Ramos, "Distributed, agent-based intelligent system for demand response program simulation in smart grids," IEEE Intelligent Systems, vol. 29, no. 1, pp. 56-65, Jan 2014.
[20] F. Fernandes, M. Silva, P. Faria, Z. Vale, C. Ramos, and H. Morais, "Real-time simulation of energy management in a domestic consumer," in IEEE PES ISGT Europe 2013, Oct 2013, pp. 1-5.

[21] Z. Vale, T. Pinto, I. Praca, and H. Morais, "Mascem: Electricity markets simulation with strategic agents," IEEE Intelligent Systems, vol. 26, no. 2, pp. 9-17, March 2011.

[22] S. Cincotti and G. Gallo, The Genoa Artificial Power-Exchange. Berlin, Heidelberg: Springer Berlin Heidelberg, 2013, pp. 348-363. [Online]. Available: https://doi.org/10.1007/978-3-642-36907-0 23

[23] H. Li, S. Member, and L. Tesfatsion, "Development of open source software for power market research: The ames test bed," Journal of Energy Markets, vol. 2, no. 2, pp. 111-128, Jan 2009

[24] V. S. Koritarov, "Real-world market representation with agents," IEEE Power and Energy Magazine, vol. 2, no. 4, pp. 39-46, July 2004.

[25] H. Morais, P. Vancraeyveld, A. H. B. Pedersen, M. Lind, H. Jhannsson, and J. stergaard, "Sospo-sp: Secure operation of sustainable power systems simulation platform for real-time system state evaluation and control," IEEE Transactions on Industrial Informatics, vol. 10, no. 4 pp. 2318-2329, Nov 2014.

[26] G. Santos, T. Pinto, H. Morais, T. M. Sousa, I. F. Pereira, R. Fernandes, I. Praa, and Z. Vale, "Multi-agent simulation of competitive electricity markets: Autonomous systems cooperation for european market modeling," Energy Conversion and Management, vol. 99, pp. 387 399, 2015. [Online]. Available: http://www.sciencedirect.com/science/ article/pii/S0196890415003969

[27] F. Fernandes, H. Morais, Z. Vale, and C. Ramos, "Dynamic load management in a smart home to participate in demand response events," Energy and Buildings, vol. 82, no. Supplement C, pp. $592-$ 606, 2014. [Online]. Available: http://www.sciencedirect.com/science/ article/pii/S0378778814006161

[28] V. M. Catterson, P. C. Baker, E. M. Davidson, S. D. J. McArthur, "An upper ontology for power engineering applications," Available: http:// sites.ieee.org/pes-mas/, April 2010, accessed: 2017-05-16.

[29] G. Santos, T. Pinto, Z. Vale, I. Praça, and H. Morais, Electricity Markets Ontology to Support MASCEM's Simulations. Cham: Springer International Publishing, 2016, pp. 393-404. [Online]. Available: https://doi.org/10.1007/978-3-319-39387-2_33

[30] E. Dodu, A. M. zbayolu, O. Benli, H. E. Akn, E. Erol, T. Atasoy, O. Gre, and . Erin, "Ontology-centric data modelling and decision support in smart grid applications a distribution service operator perspective," in 2014 IEEE International Conference on Intelligent Energy and Power Systems (IEPS), June 2014, pp. 198-204.

[31] A. Espinoza, M. Ortega, C. Fernandez, J. Garbajosa, and A. Alvarez, "Software-intensive systems interoperability in smart grids: A semantic approach," in 2011 9th IEEE International Conference on Industrial Informatics, July 2011, pp. 739-744.

[32] K. H. van Dam and J. Keirstead, "Re-use of an ontology for modelling urban energy systems," in Next generation infrastructure systems for eco-cities, Nov 2010, pp. 1-6.

[33] M. Lefrançois, J. Kalaoja, T. Ghariani, and A. Zimmermann, "SEAS Knowledge Model," ITEA2 12004 Smart Energy Aware Systems, Deliverable 2.2, 2016, $76 \mathrm{p}$.

[34] M. Lefrançois, "Planned ETSI SAREF Extensions based on the W3C\&OGC SOSA/SSN-compatible SEAS Ontology Patterns," in Proceedings of Workshop on Semantic Interoperability and Standardization in the IoT, SIS-IoT, July 2017. 